\title{
Morcegos (Mammalia, Chiroptera) capturados no Campus da Universidade Federal de Sergipe, com oito novos registros para o estado
}

\author{
Patrício Adriano da Rocha ${ }^{1,5}$, Jefferson Simanas Mikalauskas ${ }^{2}$, \\ Sidney Feitosa Gouveia ${ }^{3}$, Victor Vilas-Bôas Silveira ${ }^{4} \&$ Adriano Lúcio Peracchi ${ }^{2}$ \\ ${ }^{1}$ Programa de Pós-graduação em Ciências Biológicas (Zoologia), Departamento de Sistemática e Ecologia, \\ Universidade Federal da Paraíba - UFPB, Campus I, \\ CEP 58059-900, João Pessoa, PB, Brasil,www.gti-pe.com.br/zoologia \\ ${ }^{2}$ Laboratório de Mastozoologia, Instituto de Biologia, Universidade Federal Rural do Rio de Janeiro - UFRRJ \\ Rod. BR 465, Km 7, CEP 23890-000, Seropédica, RJ, Brasil, \\ e-mail: jsimanas@hotmail.com,aperacchi@pq.cnpq.br \\ ${ }^{3}$ Programa de Pós-graduação em Ecologia e Evolução, Instituto de Ciências Biológicas, \\ Universidade Federal de Goiás - UFG, Campus II, \\ CEP 74001-970, Goiânia, GO, Brasil,e-mail: sidneyfgouveia@superig.com.br,www.ecoevol.ufg.br \\ ${ }^{4}$ Programa de Pós-graduação em Saúde Pública e Meio Ambiente, \\ Fundação Oswaldo Cruz, Campus Sede, \\ Av. Brasil, 4365, CEP 21040-360, Manguinhos, Rio de Janeiro, RJ, Brasil, \\ e-mail: victorvbsilveira@yahoo.com.br,http://www.ensp.fiocruz.br \\ ${ }^{5}$ Autor para correspondência: Patrício Adriano da Rocha, e-mail: parocha2@yahoo.com.br
}

ROCHA, P.A., MIKAlAUSKAS, J.S., GOUVEIA, S.F., SILVEIRA, V.V.B. \& PERACCHI, A.L. Bats (Mammalia, Chiroptera) captured at the campus of the Federal University of Sergipe, including eight new records for the state. Biota Neotrop. 10(3): http://www.biotaneotropica.org.br/v10n3/en/abstract?shortcommunication+bn03310032010.

\begin{abstract}
In this study we present a list of bats recorded in an urban forest fragment at the campus of the Federal University of Sergipe, Northeastern Brazil. From July 2005 to September 2007 we collected a total of 400 individuals, distributed in five families, 13 genera and 18 species. Phyllostomidae was the most speciose family. Carollia perspicillata, Artibeus lituratus and Platyrrhinus lineatus were the most abundant species, comprising more than $65 \%$ of captured individuals. This work presents the first occurrence for eight species in Sergipe state, including one in Atlantic forest. Comments on reproductive aspects of more abundant species are included.
\end{abstract}

Keywords: chiropterans, Sergipe State, urban fragment, range, reproduction.

ROCHA, P.A., MIKALAUSKAS, J.S., GOUVEIA, S.F., SILVEIRA, V.V.B. \& PERACCHI, A.L. Morcegos (Mammalia, Chiroptera) capturados no Campus da Universidade Federal de Sergipe, com oito novos registros para o estado. Biota Neotrop. 10(3): http://www.biotaneotropica.org.br/v10n3/pt/abstract?shortcommunication+bn03310032010.

Resumo: Neste estudo apresentamos uma lista de morcegos registrados em um fragmento florestal urbano do Campus da Universidade Federal de Sergipe, Nordeste do Brasil. De julho de 2005 a setembro de 2007 coletamos um total de 400 indivíduos, distribuídos em cinco famílias, 13 gêneros e 18 espécies. Phyllostomidae foi a família com a maior riqueza de espécies. Carollia perspicillata, Artibeus lituratus e Platyrrhinus lineatus foram as mais abundantes, compreendendo mais de $65 \%$ dos indivíduos capturados. Este trabalho apresenta a primeira ocorrência de oito espécies par o Estado de Sergipe, incluindo uma para a Mata Atlântica. Observações sobre aspectos reprodutivos das espécies mais abundantes são incluídas.

Palavras-chave: quirópteros, Sergipe, fragmento urbano, área de distribuição, reprodução. 


\section{Introdução}

A identificação das faunas locais é essencial para se compreender os padrões regionais de diversidade biológica, além de permitir uma melhor caracterização da distribuição geográfica de táxons específicos (Soulé \& Wilcox 1980). A ordem Chiroptera possui cerca de 1.120 espécies, das quais $80 \%$ são exclusivas dos trópicos (Simmons 2005, Willig et al. 2003). No Brasil são conhecidas nove famílias, distribuídas em 64 gêneros e 167 espécies (Reis et al. 2007). Apesar de ser o segundo grupo de mamífero em diversidade de espécies no país, perfazendo quase um terço das espécies terrestres (Reis et al. 2007), os morcegos ainda são biológica e ecologicamente pouco conhecidos, mesmo aquelas espécies consideradas comuns (Miretzki 2003).

Sendo o menor estado brasileiro, Sergipe apresenta-se como uma lacuna de informação para a grande maioria dos grupos taxonômicos. Com relação a quirópteros, apenas o Parque Nacional Serra de Itabaiana foi razoavelmente amostrado, onde se registrou a ocorrência de 16 espécies (Mikalauskas 2005). O objetivo deste trabalho é apresentar as espécies de morcegos que ocorrem no campus Prof. José Aloísio de Campos da Universidade Federal de Sergipe e fornecer informações sobre a abundância e os aspectos reprodutivos das espécies.

\section{Material e Métodos}

\section{1. Área de estudo}

O campus da Universidade Federal de Sergipe (UFS) localizase entre os municípios de Aracaju e São Cristóvão (10 $55^{\prime} \mathrm{S}$ e $37^{\circ} 04^{\prime}$ O) (Figura 1). Por tratar-se de uma área urbana, sua vegetação original apresenta-se fortemente descaracterizada, sendo composta atualmente por grandes áreas abertas com gramíneas e árvores espaçadas entre as edificações (resultado da arborização da cidade universitária) e por um fragmento de vegetação composto por arbustos baixos, palmeiras, arvoretas e árvores de até $15 \mathrm{~m}$ de altura (Oliveira \& Lírio 2000). Dentre as espécies arbóreas das áreas abertas encontra-se Anacardium occidentale L., Coccus nucifera L., Mangifera indica L., Pachira aquatica Aubl. e Paullinia elegans Cambess. No fragmento a leste das edificações, predominam Annona sp., Cecropia sp. e Syzygium sp. Algumas regiões deste fragmento sofrem influência da expansão das águas do Rio Poxim durante a estação chuvosa (entre março e agosto), formando áreas alagadiças que se retraem no restante do ano.

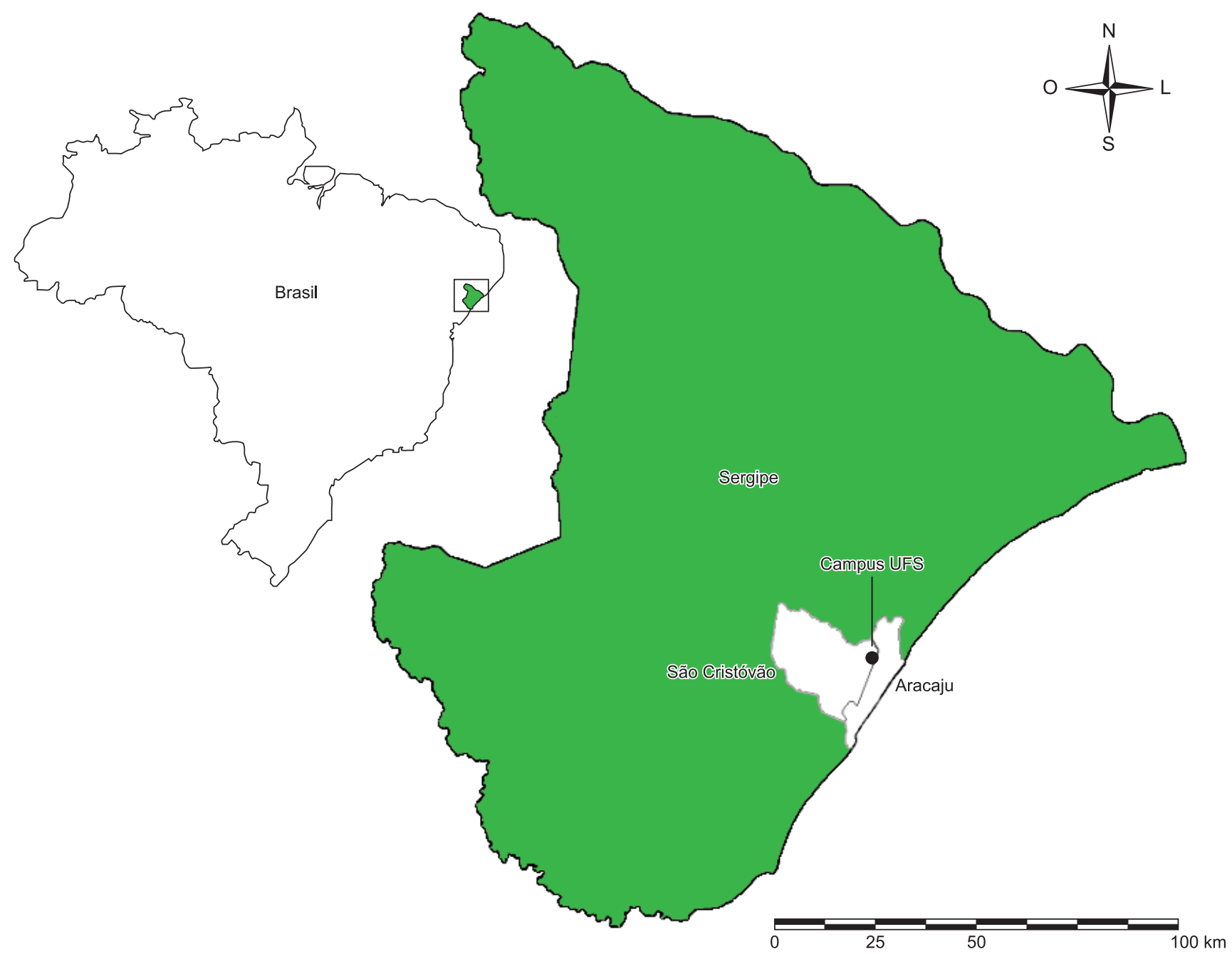

Figura 1. Localização do campus principal da Universidade Federal de Sergipe (UFS) em São Cristóvão.

Figure 1. Location of the main campus of the Federal University of Sergipe (UFS) at São Cristóvão. 


\section{Amostragem}

Entre julho de 2005 e setembro de 2007 foram realizadas 18 sessões de captura. Os animais foram coletados com o auxílio de redes de neblina armadas nas bordas e no interior do fragmento. Em cada sessão de captura, duas redes (10 e 15 m) eram estendidas entre 17:30 e 23:00 horas, sendo vistoriadas a cada 20 minutos, totalizando um esforço amostral de 5.625 h.m², conforme medida proposta por Straube \& Bianconi (2002). Foram feitas ainda cinco sessões de busca ativa, de aproximadamente duas horas cada, na área edificada do campus, na tentativa de localizar abrigos diurnos.

Após a captura dos morcegos, o sexo, o estádio reprodutivo, o peso e o comprimento do antebraço foram registrados. Posteriormente os indivíduos foram marcados com anilhas plásticas numeradas e liberados. O estádio reprodutivo foi verificado a partir da observação de caracteres sexuais secundários, segundo Fleming et al. (1972), e categorizado como: i) Fêmea prenha: quando o feto pode ser percebido no útero por apalpação; ii) Fêmea lactante: mamas intumescidas, sem pêlos e com presença de leite; iii) Fêmea prenha e lactante: presença simultânea das duas categorias anteriores e; iv) Fêmea pós-lactante: mamas sem pêlos e ausência de leite. Alguns indivíduos de cada espécie (máximo de três) foram acondicionados em sacos de pano até o fim da sessão de captura, quando foram então transportados ao laboratório, onde foram sacrificados por asfixia com uma solução de éter etílico e clorofórmio, fixados em formol a $10 \%$ e preservados em álcool 70\% para testemunho e posterior identificação. Os exemplares preservados estão sendo incorporados à Coleção Adriano L. Peracchi, Laboratório de Mastozoologia da Universidade Federal Rural do Rio de Janeiro.

A riqueza local de espécies foi estimada através do método Jackknife de $1^{\circ}$ ordem e a diversidade pelo índice de Shannon.

\section{Resultados e Discussão}

Foram capturados 400 indivíduos pertencentes a cinco famílias, 13 gêneros e 18 espécies: Phyllostomidae (12), Noctilionidae (2), Molossidae (2), Emballonuridae (1) e Vespertilionidae (1) (Tabela 1). A riqueza estimada foi de 22,53 espécies, apontando um esforço amostral satisfatório (Figura 2). O índice de diversidade de Shannon estimado foi de 2,03, sendo semelhante ao de vários outros estudos (e.g. Fazzolari-Correa 1995, Aguiar 1994, Esberard 2003). Pedro \& Taddei (1997) sugerem que embora haja mudanças na composição de espécies em diferentes ecossistemas, para morcegos o índice de diversidade em áreas neotropicais geralmente situa-se em torno de 2,0.

Dentre as espécies coletadas, Rhynchonycteris naso, Noctilio albiventris, Noctilio leporinus, Molossus molossus, Cynomops planirostris, Phyllostomus hastatus, Uroderma bilobatum e Macrophyllum macrophyllum constituem novos registros para o estado (Tabela 1). Todas essas espécies são de ampla distribuição no Brasil (Gardner 2007, Reis et al. 2006, 2007) entretanto, três delas merecem destaque. No Nordeste, M. macrophyllum só havia sido registrado para o Estado da Bahia, de modo que este registro amplia sua distribuição em aproximadamente $300 \mathrm{~km}$. A presença de Noctilio albiventris representa o primeiro registro dessa espécie para o litoral oriental do Brasil e consequentemente para a Mata Atlântica, ampliando sua distribuição em $400 \mathrm{~km}$ ao leste, quando comparado ao registro feito por Vieira (1942) em Juazeiro, BA. Uroderma bilobatum também tem a sua distribuição ampliada, uma vez que, segundo Gardner (2007), a distribuição dessa espécie na Mata Atlântica tinha seu limite norte no registro feito por Faria et al. (2006) em Ilhéus, BA, o qual é ampliado em aproximadamente $500 \mathrm{~km}$.

Os Phyllostomidae Carollia perspicillata (24\%), Artibeus lituratus $(21,8 \%)$ e Platyrrhinus lineatus $(21,5 \%)$ foram as espécies mais abundantes, perfazendo mais de $65 \%$ dos indivíduos capturados.

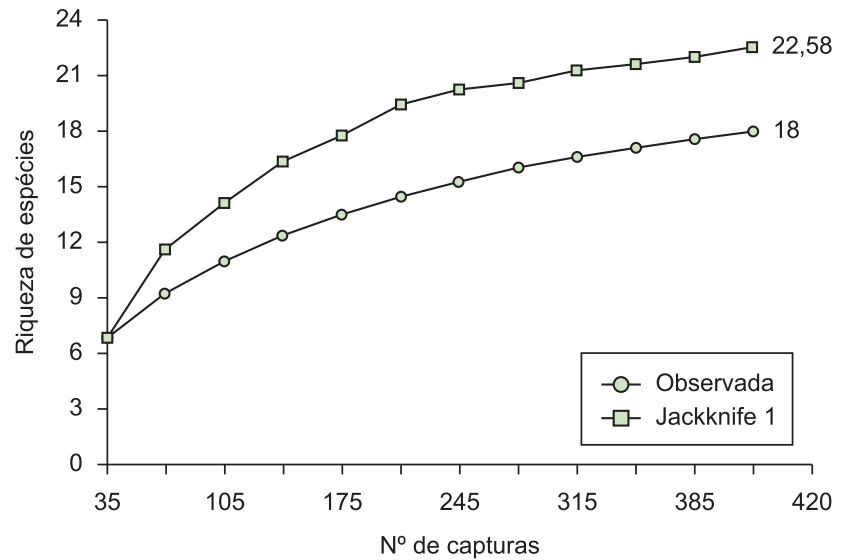

Figura 2. Curvas de acumulação observada e estimada (Jackknife 1) de espécies de morcegos do campus da UFS.

Figure 2. Observed and estimated (Jackknife 1) accumulation curves of chiropteran species from the UFS campus.

Macrophyllum macrophyllum, M. nigricans e C. planirostris tiveram apenas um indivíduo capturado (Figura 3). Myotis nigricans e C. planirostris foram capturados apenas por busca ativa em abrigos localizados em edificações da UFS. A distribuição da abundância seguiu o padrão geral encontrado para a ordem na região neotropical, com a presença de algumas espécies dominantes e muitas espécies raras (Trajano 1985, Pedro \& Taddei 1997).

A Família Phyllostomidae compreendeu mais de 95\% dos indivíduos capturados (Figura 4). Sua predominância pode ser explicada pelo fato desta ser a família mais rica na região neotropical. Além disso, o principal método de coleta empregado é seletivo, favorecendo a captura de espécies frugívoras de vôo mais baixo em detrimento de insetívoras, como Emballonuridae, Molossidae, Mormoopidae e Vespertilionidae, as quais voam em estratos mais elevados e detectam as redes com maior facilidade (Arita 1993, Pedro \& Taddei 1997, Passos et al. 2003, Peracchi et al. 2006). É possível ainda que as atividades humanas favoreçam a expansão de espécies frugívoras generalistas, que passam a contar com novas fontes de alimento, representadas pelas plantações e pelo grande número de plantas frutíferas que arborizam as cidades (Trajano 1985). Isso explicaria a predominância de espécies como A. lituratus, C. perspicillata e P. lineatus.

A presença de fêmeas prenhas de $C$. perspicillata capturadas de janeiro a setembro (Tabela 2) demonstra a plasticidade ecológica da espécie diante da disponibilidade de recursos. Ao lado dos Stenodermatinae A. fimbriatus e A. lituratus, a espécie apresenta um curto período de inatividade reprodutiva, concordando com as categorias de padrões reprodutivos dos morcegos neotropicais definidas por Wilson (1973, 1979). Alguns estudos (ex. Zortéa 2003, De Kengt et al. 2005, Silva 2007, Ortêncio Filho et al. 2007) apontam a relação entre atividade reprodutiva dos morcegos frugívoros e o período de maior disponibilidade de recursos alimentares, o que favoreceria a sobrevivências das proles. $\mathrm{Na}$ área de estudo, atribuímos o fato à presença de um grande número de árvores frutíferas (mangueiras, cajueiros, goiabeiras e andirobas) introduzidas na área do campus, as quais proporcionam um aporte de recursos suficiente para a ampliação do período reprodutivo dessas espécies. Adicionalmente, a presença de fêmeas de $A$. cinereus, A. fimbriatus e A. obscurus simultaneamente prenhas e lactantes indicam a presença de estro pós-parto. A Tabela 2 trás ainda informações pontuais para algumas espécies. 
Tabela 1. Morcegos do campus José Aloísio de Campos da Universidade Federal de Sergipe, São Cristóvão, Sergipe, acompanhados dos registros nos estados brasileiros, segundo Reis et al.(2006, 2007) adicionado de Mikalauskas (2005). Asteriscos indicam os primeiros registros para Sergipe.

Table 1. Bats from the campus José Aloísio de Campos of the Federal University of Sergipe, São Cristóvão, Sergipe, followed by the records along Brazilian states, according to Reis et al.(2006, 2007) added of Mikalauskas (2005). Asterisks indicate the first record for Sergipe State.

\begin{tabular}{lccccc}
\hline \multirow{2}{*}{ Espécie } & \multicolumn{4}{c}{ Região } \\
\cline { 2 - 6 } & Centro-oeste & Nordeste & Norte & Sudeste & Sul
\end{tabular}

Família Emballonuridae

Rhynchonycteris naso* (Weid, 1820)

Familia Noctilionidae

Noctilio leporinus* (Linnaeus, 1758)

Noctilio albiventris* (Linnaeus, 1758)

Família Phyllostomidae

Subfamília Phyllostominae

Macrophyllum macrophyllum* (Scinz, 1821)

Phyllostomus discolor (Wagner, 1834)

Phyllostomus hastatus* (Thomas, 1901)

Subfamília Glossophaginae

Glossophaga soricina (Pallas, 1766)

Subfamília Carollinae

Carollia perspicillata (Linnaeus, 1758)

Subfamília Stenodermatinae

Sturnira lilium (E. Geoffroy,1810)

Platyrrhinus lineatus ( E. Geoffroy, 1810)

Artibeus cinereus (Gervais, 1855)

Artibeus fimbriatus (Gray, 1838)

Artibeus lituratus (Olfers, 1818)

Artibeus obscurus (Schinz, 1821)

Uroderma bilobatum* (Peters, 1866)

Família Molossidae

Cynomops planirostris* (Peters, 1866)

Molossus molossus* (Pallas, 1766)

Família Vespertilionidae

Myotis nigricans (Schinz, 1821)
GO, MT

AL, BA, PE, PI, SE

GO, MS, MT

BA, CE, PB, PE, SE

MS, MT

BA, CE, PI SE

GO

BA, SE

AC, AM, AP

DF, MS, MT

BA, CE, PB, PE, PI, SE

AC, A RR

, AP,

AC, AM, AP, PA, RO, RR PE, PI, SE MT

DF, GO, MS, MT

BA, CE, MA, $\mathrm{PB}, \mathrm{PE}, \mathrm{PI}, \mathrm{SE}$

AC, AM, AP, PA, RO, RR

ES, MG, RJ, PR, RS, SC SP

DF, GO, MS $\mathrm{MT}$

AL, BA, CE,
PB, PE, PI, SE

AC, AM, AP, MA, PA, RO, RR

ES, MG, RJ SP

DF, MS, MT

$\mathrm{AL}, \mathrm{BA}, \mathrm{CE}$,

AC, AM, AP,

$\mathrm{PB}, \mathrm{PE}, \mathrm{SE}$

PA, RO, RR

DF, GO, MS

BA, CE, PB, MT

PE, PI, SE

DF, MT

AL, BA, MA, PB, PE, SE

AC, AM, AP,

MA, PA, RO

DF

BA, CE, PE,

SE

DF, MS, MT

AL, BA, CE, PE, SE

AC, AM, AP, PA, RO, RR

MT

BA, CE, PB, PE, PI, SE

AC, AM, AP, PA, RO, RR

BA, SE

AC, AM, AP, PA, RO, RR

MS, MT

BA, PE, SE

AM, PA

ES, MG, SP

PR

DF, MS, MT

BA, CE, MA, PE, SE

$\mathrm{AC}$, RO, RR

ES

ES, MG, RJ, PR, RS, SC SP

DF,GO,MS MT

ES, MG, RJ, PR, RS, SC SP 
Tabela 2. Estágio reprodutivo das fêmeas (número de indivíduos entre parênteses) de espécies ao longo dos meses do ano entre 2005 e 2007 no campus da Universidade Federal de Sergipe. Ausência de dados é mantida em branco a título de clareza. P = Prenha; L = Lactante; PL = Prenha e Lactante; F = Fêmea com filhote; Pós-L = Pós-Lactante.

Table 2. Female reproductive stage (number of individuals in parenthesis) of the species along the months between 2005 and 2007 at the campus of Federal University of Sergipe. Data absence is left in blank for clarity. P = Pregnant; $\mathrm{L}=$ Lactating; PL = Pregnant and Lactating; F = Female with young; Pós-L = PostLactating.

\begin{tabular}{|c|c|c|c|c|c|c|c|c|c|c|c|c|}
\hline Espécie & Jan. & Fev. & Mar. & Abr. & Maio & Jun. & Jul. & Ago. & Set. & Out. & Nov. & Dez. \\
\hline R. naso & - & - & - & - & - & - & & $\mathrm{P}(1)$ & - & - & - & - \\
\hline N. leporinus & - & - & - & - & - & - & - & & - & $\begin{array}{l}\mathrm{P}(2), \\
\mathrm{L}(1)\end{array}$ & - & - \\
\hline N. albiventris & - & - & - & - & - & - & - & $\mathrm{L}(1)$ & - & - & - & - \\
\hline G. soricina & $\mathrm{P}(1)$ & - & - & - & - & - & - & - & - & - & - & - \\
\hline C. perspicillata & $\begin{array}{c}\text { P(1), L(1), } \\
\text { Pós-L(1), } \\
\text { F(1) }\end{array}$ & - & $\mathrm{P}(1)$ & $\mathrm{P}(1)$ & $\mathrm{P}(2), \mathrm{L}(2)$ & $\begin{array}{c}\mathrm{L}(2), \\
\text { Pós-L(2), } \\
\mathrm{F}(1)\end{array}$ & $\begin{array}{c}\text { P(2), } \\
\text { Pós-L(3) }\end{array}$ & - & $\mathrm{P}(2)$ & - & - & - \\
\hline S. lilium & - & - & $\mathrm{P}(1)$ & - & - & $\mathrm{P}(1)$ & - & - & - & - & - & - \\
\hline P. lineatus & $\begin{array}{l}\text { P(2), L(1), } \\
\text { Pós-L(4) }\end{array}$ & - & $\mathrm{P}(2)$ & $\mathrm{P}(2), \mathrm{L}(3)$ & $\begin{array}{c}\text { L(3), } \\
\text { Pós-L(3) }\end{array}$ & $\begin{array}{c}\text { L(3), } \\
\text { Pós-L(4) }\end{array}$ & - & - & - & - & - & - \\
\hline A. cinereus & $\mathrm{P}(3), \mathrm{PL}(1)$ & - & - & - & - & - & - & $\mathrm{P}(1)$ & - & - & - & - \\
\hline A. fimbriatus & $\mathrm{P}(3), \mathrm{PL}(1)$ & - & $\mathrm{L}(1)$ & $\begin{array}{c}\text { L(1), } \\
\text { Pós-L(3) }\end{array}$ & - & Pós-L(1) & - & - & $\mathrm{P}(1)$ & - & - & - \\
\hline A. lituratus & $\mathrm{P}(1)$ & - & $\mathrm{L}(1)$ & $\begin{array}{c}\mathrm{L}(1), \\
\text { Pós-L(1) }\end{array}$ & $\mathrm{P}(2), \mathrm{L}(1)$ & $\begin{array}{c}\text { P(2), } \\
\text { Pós-L(1) }\end{array}$ & $\mathrm{P}(3)$ & $\mathrm{P}(1)$ & $\mathrm{P}(1)$ & - & $\begin{array}{l}\mathrm{P}(1), \\
\mathrm{L}(1)\end{array}$ & - \\
\hline A. obscurus & - & - & - & Pós-L(1) & $\begin{array}{c}\text { PL(2), } \\
\text { Pós-L(2) }\end{array}$ & - & $\mathrm{P}(1)$ & Pós-L(2) & - & - & - & - \\
\hline U. bilobatum & - & - & - & - & - & Pós-L(1) & - & - & - & - & - & - \\
\hline C. planirostris & - & - & $\mathrm{L}(1)$ & - & - & - & - & - & - & - & - & - \\
\hline
\end{tabular}

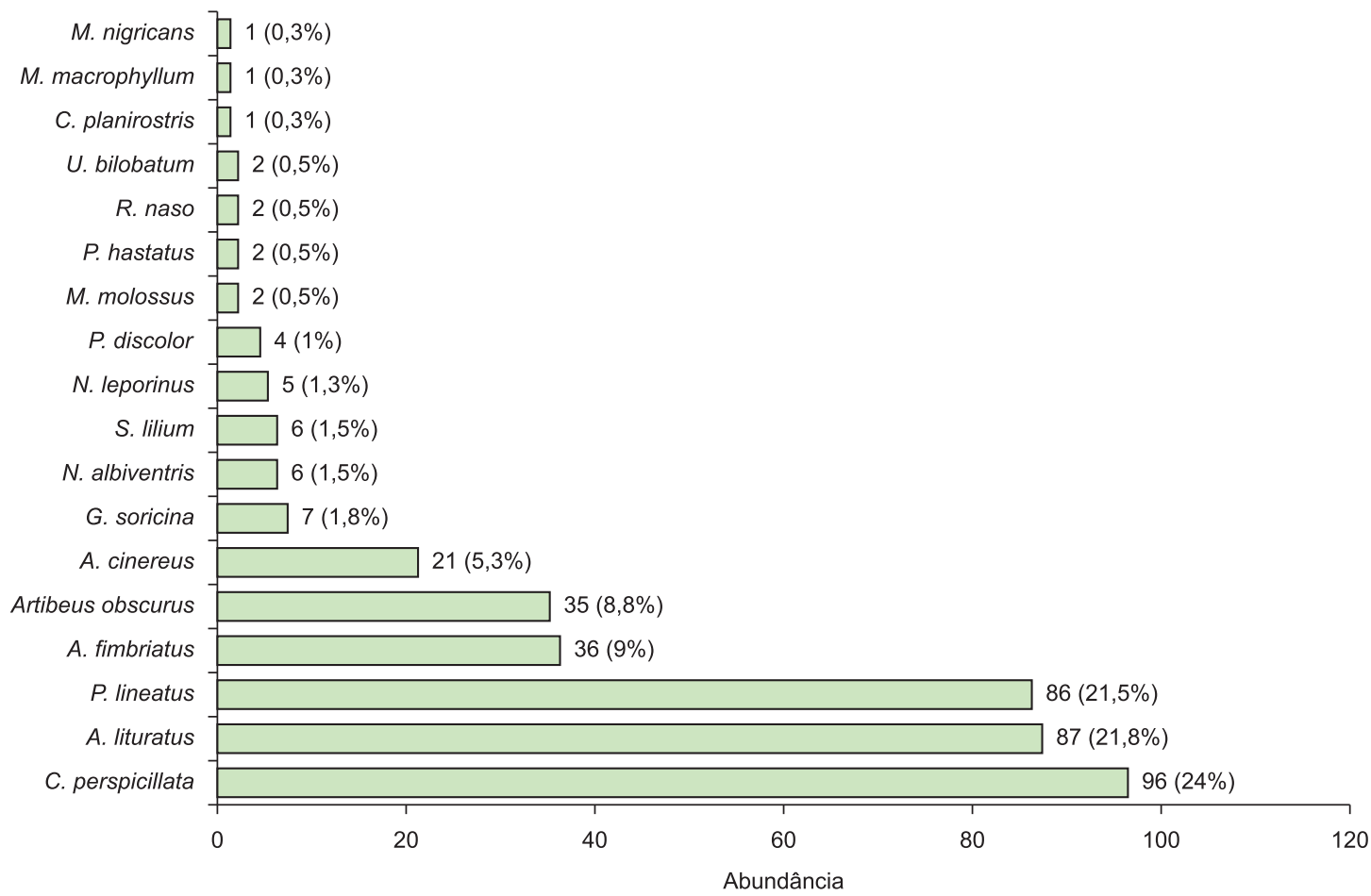

Figura 3. Abundância absoluta das espécies de morcegos do campus da UFS.

Figure 3. Absolute abundance of bat species from the UFS campus. 


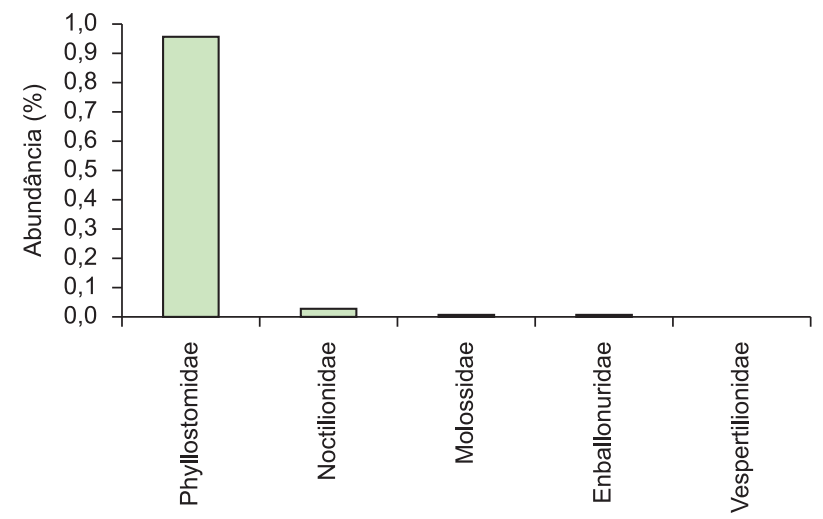

Figura 4. Abundância relativa de famílias de morcegos do campus da UFS. Figure 4. Relative abundance of bat families from the UFS campus.

De forma geral, o conhecimento sobre a quiropterofauna sergipana é ainda bastante incipiente. Considerando as espécies apresentadas por Alencar et al. (1994), Mikalauskas (2005) e por Feijó \& Nunes (2010), somadas às registradas no presente trabalho, são 27 espécies conhecidas até o momento. Certamente estudos futuros em outras localidades ampliarão significativamente a riqueza de espécies para o estado e poderão contribuir no reconhecimento dos padrões de distribuição geográfica dos morcegos, bem como dos seus papéis funcionais dentro dos ecossistemas de que fazem parte.

\section{Agradecimentos}

Somos gratos a Stephen Ferrari pela leitura crítica do manuscrito, a Carla Simonini Bastos pelo auxílio nos trabalhos de campo e à equipe de vigilância do campus da UFS pela segurança nos trabalhos noturnos.

\section{Referências Bibliográficas}

AGUIAR, L.M.S. 1994. Comunidades de Chiroptera em três áreas de Mata Atlântica em diferentes estádios de sucessão. Estação Biológica de Caratinga, Minas Gerais. Dissertação de Mestrado, Universidade Federal de Minas Gerais, Belo Horizonte.

AlENCAR, A.O., SIlVA, G.A.P., ARRUdA, M.M., SOARES, A.J. \& GUERRA, D.G. 1994. Aspectos biológicos e ecológicos de Desmodus rotundus (Chiroptera) no nordeste do Brasil. Pesq. Vet. Bras. 14(4):95-103.

ARITA, H.T. 1993. Rarity in neotropical bats: correlations with phylogeny, diet and body mass. Ecol. App. Ann. Arbor. 3(3):506-517.

DE KNEGT L.V.; SILVA J.A.; MOREIRA E.C. E SALES G.L. 2005. Morcegos capturados no Município de Belo Horizonte, 1999-2003. Arquivo Brasileiro de Medicina Veterinária e Zootecnia 57: 576-583.

ESBÉRARD, C.E.L. 2003. Diversidade de morcegos em área de Mata Atlântica regenerada no sudeste do Brasil. Rev. Bras. Zoocienc. 5(2):189-204.

FARIA, D., SOARES-SANTOS, B. \& SAMPAIO, E. 2006. Bats from the Atlantic rainforest of southern Bahia, Brazil. Biota Neotrop. 6:1-13.

FAZZOLARI-CORREA, S. 1995. Aspectos sistemáticos, ecológicos e reprodutivos de morcegos na Mata Atlântica. Tese de Doutorado, IB, Universidade de São Paulo, São Paulo.

FEIJÓ, J.A. \& NUNES, H.L. 2010. Mammalia, Chiroptera, Phyllostomidae, Artibeus planirostris (Spix, 1823) and Trachops cirrhosus (Spix, 1823): First record for the state of Sergipe, northeastern Brazil. Check List. 6(1):15-16.

FLEMING, T.H., HOOPER, E.T. \& WILSON, D.E. 1972. Three Central American bat communities: structure, reproductive cycles, and movement patterns. Ecology 53:555-569.
GARDNER, A.L. 2007. Mammals of South America, Vol. 1: marsupials, xenarthrans, shrews, and bats. University of Chicago Press, Chicago.

MIKALAUSKAS, J.S. 2005. Morcegos. In Parque Nacional Serra de Itabaiana - Levantamento da Biota (C.M. Carvalho \& J.C. Vilar, Coord.). IBAMA, UFS, Aracaju,SE, p.93-103. Biologia Geral e Experimental.

MIRETZKI, M. 2003. Morcegos do Estado do Paraná, Brasil (Mammalia, Chiroptera): riqueza de espécies, distribuição e síntese do conhecimento atual. Pap. Av. Zool. 43(6):101-138.

OLIVEIRA, F.F. \& LÍRIO Jr., G.P. 2000. Anfíbios anuros do Campus da Universidade Federal de Sergipe. Rev. Biol. Ger. Experim. 1(1):42-74.

ORTÊNCIO FILHO, H; REIS, N. R.; PINTO, D \& VIEIRA, D. C. 2007. Aspectos reprodutivos de Artibeus lituratus (Phyllostomidae) em fragmentos florestais na região de Porto Rico, Paraná, Brasil. Chiropt. Neotrop. 13 (2): 313-318.

PASSOS, F.C., SILVA, W.R., PEDRO, W.A. \& BONIN, M.R. 2003. Frugivoria em morcegos (Mammalia, Chiroptera) no Parque Estadual Intervales, Sudeste do Brasil. Rev. Bras. Zool. 20(3):511-517.

PEDRO, W.A. \& TADDEI, W.A. 1997. Taxonomic assemblage of bats from Panga Reserve, Southeastern Brazil: abundance patterns and trophic relations in the Phyllostomidae (Chiroptera). Bol. Mus. Biol. Mello Leitão 6:3-21.

PERACCHI, A.L., LIMA, I.P., REIS, N.R., NOGUEIRA, M.R. \& FILHO, H.O. 2006. Ordem Chiroptera. In Mamíferos do Brasil (N.R. Reis, A.L. Peracchi, W.A. Pedro \& I.P. Lima, Ed.). EDIFURB, Londrinha, p.153-230.

REIS, N.R., PERACCHI, A.L., PEDRO, W.A. \& LIMA, I.P. (Org.). 2006. Mamíferos do Brasil. EDIFURB, Londrina, 437p.

REIS, N.R., PERACCHI, A.L., PEDRO, W.A. \& LIMA, I.P. (Org.). 2007. Morcegos do Brasil. EDUEL, Londrina, 252p.

SILVA, L. A. M.. 2007. Comunidades de morcegos na caatinga e brejo de altitude, no agreste de Pernambuco. Dissertação de mestrado. Universidade Federal de Brasília.

SIMMONS, N.B. 2005. Order Chiroptera. In Mammal species of the World: a taxonomic and geographic reference. $3^{\text {th }}$ ed. v. 1. (D.E. Wilson \& D.M. Reeder, Ed.). Johns Hopkins University Press, p.312-529.

SOULÉ, M.E. \& WILCOX, B.A. 1980. Conservation Biology: its scope and its challenge. In Conservation Biology (M.E. Soulé \& B.A. Wilcox, Ed.). Linauer Associates, Massachussetts, p. 1-8.

STRAUBE, F.C. \& BIANCONI, G.V. 2002. Sobre a grandeza e a unidade utilizada para estimar esforço de captura com utilização de redes de neblina. Chiropt. Neotrop. 8(1-2):150-152.

TRAJANO, E. 1985. Ecologia de populações de morcegos cavernícolas em uma região cárstica do sudeste do Brasil. Rev. Bras. Zool. 2(5):255-320.

WILLIG, M.R., KAUFMAN, D.M. \& STEVENS, R.D. 2003. Latitudinal gradients of biodiversity: pattern, process, scale and synthesis. Ann. Rev. Ecol. Syst. 34:237-309.

WILSON, D.E. 1973. Reproduction in Neotropical bats. Period. Biol. 75:215-217.

WILSON, D.E. 1979. Reproductive patterns. In Biology of bats of the New World family Phyllostomatidae. Part III. (R.J. Baker, J.K. Jones Jr. \& D.C. Carter, Ed.). Museum of Texas Tech Univ, p.317-378.

ZORTÉA, M. 2003. Reproductive Patterns and Feeding habits of three nectarivorous bats (Phyllostomidae: Glossophaginae) from the Brazilian Cerrado, Brazil. J. Biol., 63(1): 159-168. 